\title{
An atypical cause of nausea and vomiting during pregnancy
}

\begin{abstract}
Nausea and vomiting during the first trimester of pregnancy are frequent and, usually, self-limited. We report the case of a woman diagnosed with nausea and vomiting associated with pregnancy. On a second emergency episode in which she complained of nausea, vomiting and frontal headache, she was hospitalized. The same day she had seizures and was diagnosed with a cerebral neoplasia. She died few hours later. Careful assessment is required when a pregnant woman complains of nausea and vomiting. Multiple diagnosis and complications should be sought, particularly if there atypical signs or symptoms.
\end{abstract}

Volume 7 Issue 5 - 2018

\author{
Ana Edral,' Rita Martins,' Fernanda Vilela,' \\ Carolina Oliveira, ${ }^{2}$ and Ângela Ferreira ${ }^{2}$ \\ 'Department of Obstetrics and Gynecology Resident, Centro \\ Hospitalar do Algarve, Portugal \\ ${ }^{2}$ Department of Obstetrics and Gynecology Specialist, Centro \\ Hospitalar do Algarve, Portugal
}

\begin{abstract}
Correspondence: Ana Edral, Department of Obstetrics and Gynecology Resident, Centro Hospitalar Universit $\tilde{A}_{j}$ rio do Algarve, Rua LeÃfo Penedo 8000-386 Faro, Portugal,Tel
\end{abstract}

912642783, Email aebral@chalgarve.min-saude.pt

Received: April 0I, 2018 | Published: November 17, 2018

\section{Introduction}

Nausea and vomiting are common symptoms in pregnancy, affecting about $50-80 \%$ of pregnant women. ${ }^{1}$ It affects mostly women in the first trimester of pregnancy, starting around 4-6 weeks and peaking between 8-12 weeks. The symptoms fade away around 20th gestational week. ${ }^{2,3}$ The most severe form is called hyperemesis gravidarum. It occurs in $0,5-3 \%$ of pregnant women and presents with incoercible vomiting, weight loss exceeding $5 \%$ of the pre-pregnancy weight, dehydration, ketonuria, hypokalemia. ${ }^{2,3}$ However, when facing a pregnant patient complaining of vomiting, the diagnosis of vomiting associated with pregnancy or hyperemesis is one of exclusion. ${ }^{4}$ When there are atypical signs and/or symptoms, there should be a more indepth examination, in order to exclude other causes and determine if they are associated with pregnancy or not (Table 1).

Table I Some examples of differential diagnosis of nausea and vomiting during pregnancy

\section{Differential diagnosis of Nausea and Vomiting in pregnancy ${ }^{4,5}$}

$\begin{array}{ll}\text { Gastrointestinal } & \begin{array}{l}\text { Gastroparesis, gastroenteritis, gastro-esophageal } \\ \text { reflux, peptic ulcer, diaphragmatic hernia, hepatitis, }\end{array} \\ \text { pathology } & \text { biliary tract disease, inflammatory bowel disease, } \\ & \text { pancreatitis, appendicitis, intestinal occlusion }\end{array}$

$\begin{array}{ll}\text { Genital-Urinary } & \text { Acute pyelonephritis, uremia, ovarian cyst torsion, } \\ \text { pathology } & \text { renal lithiasis }\end{array}$

Metabolic

Pathology

Neurological pathology

Metabolic ketoacidosis, hyperthyroidism

Vestibular lesions, CNS tumors, intracranial hypertension, migraines

Diseases of pregnancy

Hyperemesis gravidarum, gestational trophoblastic disease, multiple pregnancy, acute fatty liver of pregnancy, preeclampsia, placental abruption

Other Intoxication, psychogenic/psychiatric, drugs, neoplasia

\section{Clinical case}

A patient with unremarkable medical individual or familial history, except for a history of Raynaud's Syndrome, presented in the emergency service (ER) of our institution. She complained of headache, nausea and vomiting, with 3 days of evolution. The physical examination was normal (including the neurological examination). The blood analysis revealed $\beta$-hCG value of $15000 \mathrm{mU} / \mathrm{mL}$ (nonpregnant reference value $<5 \mathrm{mU} / \mathrm{mL}$ ), therefore she was referred to the Obstetrics ER. In her obstetrical past history, she had had 2 uneventful pregnancies, with term deliveries. The patient couldn't recall her last menstruation. The gynecological examination was normal and the pelvic ultrasound revealed a single intrauterine pregnancy of about 6-7 weeks. The patient was discharged to her attending physician (general practitioner-GP).

A month later, she returned to the Obstetrics ER for frequent vomiting. She had signs of widespread malaise, excessive sweating and pallor; she was conscious, cooperative, oriented in time and space. Her blood analysis was normal. However, she was admitted to the Obstetrics department for surveillance and symptomatic therapy. The same day, she had an unwitnessed loss of sphincter control in bed. The patient remained slightly prostrate during that afternoon. At night, she had a generalized tonic-clonic seizure (GTC), which lasted 2 minutes. She was stabilized and the internal medicine colleague was called. Neurological examination was performed: no spontaneous eye opening, imperceptible speech, but able to localize pain ( 8 score on the Glasgow Coma Scale). She was at all times hemodynamically stable. In arterial blood gas analysis, there was a metabolic acidosis with hyperlactacidemia and hypokalemia. Replacement therapy was carried out and phenytoin was initiated. Minutes later, she had a new GTC seizure and collaboration of the Intensive Care Unit (ICU) was requested. Also, it was requested a cerebral CT-scan. During the CT-scan, the patient had a cardio-respiratory arrest. Advanced life support was initiated and heart rate was recovered. After this episode, she was transferred to the ICU unit. On the CT-scan, there was an expansive right frontal-basal lesion with hemorrhagic areas and an extensive cystic area, causing a mass effect with compression of the 
right frontal lateral ventricle horn, slight midline shift and cerebral cistern deletion, and also an extra-axial frontal-basal collection predominantly hypodense (Figure 1). Pharmacological measures to reduce brain swelling and analgesia were instituted. Approximately 10 hours later the patient died. Her family refused an autopsy.

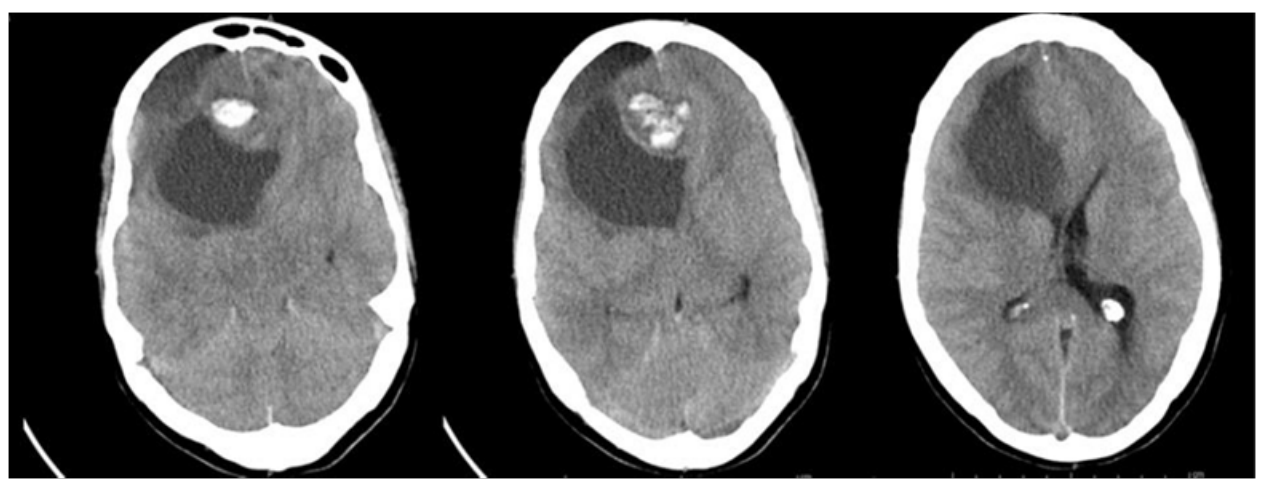

Figure I Cerebral CT-scan images (see main text for details).

\section{Discussion}

Hiperemesis gravidarum and infectious gastrointestinal diseases are the most common causes of nausea and vomiting in first trimester of pregnancy. However, we must take into account the neurological/ neurosurgical complications that can arise in this clinical context. The most common neurologic complications in pregnancy are those secondary to arterial-venous malformations, aneurysms and preeclampsia. ${ }^{5}$ Brain tumors, however, are rare and, often, their diagnosis is delayed by the similarity of its symptoms with those of a normal pregnancy or its complications, such as headaches, nausea, vomiting, visual disturbances (due to mass effect). ${ }^{6}$ The nausea and vomiting associated with normal pregnancy tend to improve later in pregnancy, on the opposite those associated with brain tumors, may arise later, tend to worsen gradually, ${ }^{7}$ and may coexist with symptoms such as headache, visual disturbances or focal neurological deficits. ${ }^{8}$ Primary intracranial tumors are the fifth cause of cancer-related death in women from 20 to 39 years. ${ }^{6}$ It is known that pregnancy does not alter the incidence of brain tumors and their prevalence reflects the same age group in the general population. ${ }^{6,8}$ However, hemodynamic and hormonal changes during the pregnancy may accelerate tumor growth at this stage. ${ }^{4,8}$ with the possible worsening of the signs and symptoms. ${ }^{7}$ In the case described, the nausea and vomiting referred by the pregnant woman were interpreted as a consequence of the pregnancy, given their early appearance. The headache, in the presence of a normal neurological examination, was interpreted as idiopathic. However, if we analyze it now, the following complaints could have been interpreted as signs of alarm: the persistence/ worsening of the headaches, the fact that the patient told us later that she felt anxious and with marital problems, and also, when she was hospitalized, one of her relatives reported that she was not interested in her domestic and family care anymore, and also showing some signs of apathy. The diagnosis of brain lesions is best achieved with MRI or CT scan., ${ }^{4,6}$ Here, we performed the CT scan because it was the only one immediately available. After the diagnosis of a brain tumor, the approach varies according to the gestational age, the maternal and fetal status and the type of tumor. The challenge is greater because there is a need to balance and optimize maternal and fetal outcomes. When it is diagnosed during the first trimester, the treatment should be delayed, if there is maternal stability and absence of symptoms. The surgical treatment is performed in the presence of malignant neoplasia and severe symptoms and it is usually postponed until the second trimester. ${ }^{4,8}$ The symptomatic treatment with corticosteroids and anticonvulsants may be necessary. ${ }^{8}$ Regarding the type of tumor, in the general population, the most frequent are the glioma (38\%) and meningioma $(28 \%) .^{6}$ There is no particular association of brain metastases to pregnancy, with the exception of choriocarcinoma. Meningioma, vestibular schwanoma and pituitary tumors may increase in volume during pregnancy and therefore become symptomatic. ${ }^{8}$ In this case, her family refused the execution of an autopsy, rendering it impossible to determine the histology of the tumor. This case displays the importance of a careful and critical approach to every pregnant woman. One should consider several differential diagnoses and also to pay attention to the atypical signs and symptoms. The multidisciplinary discussion is also of crucial importance. In the case of brain tumors, early diagnosis can make a difference in the prognosis.

\section{Acknowledgements}

None.

\section{Conflicts of interest}

Authors declare that there is no conflict of interest.

\section{References}

1. Matthews A, Haas DM, O Mathuna DP, et al. Interventions for nausea and vomiting in early pregnancy. The Cochrane database of systematic reviews. 2010;8(9):CD007575.

2. Lacasse A, Rey E, Ferreira E, et al. Nausea and vomiting of pregnancy: what about quality of life? BJOG: an international journal of obstetrics and gynaecology. 2008;115(12):1484-1493.

3. Lee NM, Saha S. Nausea and vomiting of pregnancy. Gastroenterology clinics of North America. 2011;40(2):309-334.

4. Van Calenbergh SG, Poppe WA, Van Calenbergh F. An intracranial tumouran uncommon cause of hyperemesis in pregnancy. European journal of obstetrics, gynecology, and reproductive biology. 2001;95(2):182-183.

5. Anderson WD, Strayer SM. Evaluation of Nausea and Vomiting in Adults: A Case-Based Approach. Am Fam Physician. 2013;15;88(6):371-379. 
6. Ravindra VM, Braca JA, Jensen RL, et al. Management of intracranial pathology during pregnancy: Case example and review of management strategies. Surgical Neurology International. 2015;6:43.

7. Tharian S, George N, Tharian B, et al. Hyperemesis Gravidarum: An Out of the Box Presentation, a Rare Case Report and Literature Review. J Clin Gynecol Obstet. 2014;3(4):143-146.
8. Lee MJ, Hickenbottom S. Neurologic disorders complicating pregnancy. In: Post TW, Waltham MA, editors. 2015. 